\title{
ESTIMATION OF BOOSTER KICKER IMPEDANCES
}

\section{AD \\ BOOSTER TECHNICAL NOTE \\ NO. 145}

M. RHOADES-BROWN

JULY 6, 1989

ACCELERATOR DEVELOPMENT DEPARTMENT BROOKHAVEN NATIONAL LABORATORY

UPTON, NEW YORK 11973 


\section{Estimation of Booster Kicker Impedances}

\section{Rhoades-Brown}

\section{Statement of the Problem}

The booster kicker magnets are long box-like structures ${ }^{1}$ made of ferrite with copper coils covering the inner vertical surfaces. At the present time, there are no plans to insert a copper barrier in the ferrite path at the top and bottom of the centerplate. If $2 a$ and $2 \mathrm{~b}$ represent the inner height and width respectively, the four injection kickers and six ejection kickers are distributed as follows: ${ }^{1}$

\section{Number}

4 (Injection)

2 (Ejection)

2 (Ejection)

2 (Ejection)
Inner Width (2b)

$$
4.25^{\prime \prime}
$$

$4.27^{\prime \prime}$

$5.07^{\prime \prime}$

$4.27^{\prime \prime}$
Inner Height (2a)

2.75"

$2.65^{\prime \prime}$

$2.65^{\prime \prime}$

$2.65^{\prime \prime}$
Length $(\ell)$

$5.99^{\prime \prime}$

$21.5^{\prime \prime}$

$17.9^{\prime \prime}$

$16.0^{\prime \prime}$

Of particular interest in this note is the effect of these box like structures on the beam. This is calibrated through the complex impedance $\mathrm{Z}=\mathrm{ReZ}+\mathrm{i} \operatorname{ImZ}$, which is a direct measure of beam stability. Previous calculations ${ }^{2}$ show very clearly that for the Booster, the largest contribution to the coupling impedance is the "space charge". The purpose of this note is to estimate the impedances for the kicker magnets, and to compare these numbers with the previously calculated values for the "space charge" and other accelerator components of the Booster.

To estimate the impedances, the formulation of Nassibian and Sacherer ${ }^{3}$ was used. This formulation, and subsequent developments have been summarized by $\mathrm{Ng}^{4}$ and used to estimate kicker impedance at the $\mathrm{SSC}^{5}$ and $\mathrm{RHIC}^{6}$. Using this formulation we can write,

$$
\begin{gathered}
\operatorname{Re} \frac{Z_{/ /}}{n}=\frac{\ell \nu \omega_{0}}{2 Z_{s}}\left(\frac{\mu_{0} x}{a}\right)^{2} \frac{\sin ^{2} k \ell / 2}{k \ell / 2} \Omega \\
\operatorname{Im} \frac{Z_{/ l}}{n}=\operatorname{Re} \frac{Z_{/ /}}{n} \frac{(k \ell-\sin k \ell)}{2 \sin ^{2} k \ell / 2} \quad \Omega \\
\operatorname{Re} Z_{\perp}=\frac{Z_{0} \ell}{2 a b k \ell}(1-\cos k \ell) \quad \Omega / m \\
\operatorname{Im} Z_{\perp}=\frac{Z_{0} \ell}{2 a b}\left(1-\frac{\sin k \ell}{k \ell}\right) \quad \Omega / m
\end{gathered}
$$

where $Z_{0}=377 \Omega, \omega_{0}$ is the rotation frequency, $Z_{s}$ is the line impedance taken to be $14 \Omega^{7}$, $\mu_{0}=4 \pi \times 10^{-7} \mathrm{Hm}^{-1}$ and $k=2 \pi f / \nu$ where $f$ is the value of the frequency at which the impedance is to be calculated. The phase velocity $\nu$ is given by the expression ${ }^{4}$ 


$$
\nu=\frac{Z_{s} a c}{Z_{0} b}
$$

where $c$ is the velocity of light.

The possible orbit offset $x$, in equations (1) and (2), represents the horizontal deviation of the beam from the geometric center of the box. When $x=0$, both $\operatorname{Re} Z_{/ /}$and $\operatorname{Im} Z_{/ /}$ are zero for a window-type magnet.

\section{Universal Curves for Impedance of Booster Kickers}

The Booster will accelerate both protons and several species of heavy ions. Hence, the rotation frequency $\omega_{0}$ will vary substantially for these species. In addition, the particle orbit offset $x$ will vary during the accelerator lifetime. For these reasons, I propose rewriting equations (1) - (4) in such a way that a universal character for the impedance is evident, and thus these quantities may be readily extracted for any $\omega_{0}$ or $x$. Noting $\omega_{0}=2 \pi \beta c / C$, where $\beta$ is the velocity of the ion in the Booster, and $C$ is the Booster circumference, (1) - (4) can be rewritten as,

$$
\begin{aligned}
& R e \frac{Z_{/ /}}{n}=\frac{c^{3} \mu_{0}^{2} Z_{s}}{2 b^{2} Z_{0}^{2} C} \beta x^{2} \frac{(1-\cos 2 \pi f \ell / \nu)}{f} \Omega \\
& \equiv F(f) \beta x^{2} \quad \Omega \\
& \operatorname{Im} \frac{Z_{/ /}}{n}=F(f) \beta x^{2} \frac{(k \ell-\sin k \ell)}{2 \sin ^{2} k \ell / 2} \quad \Omega \\
& \equiv G(f) \beta x^{2} \quad \Omega
\end{aligned}
$$

Also,

$$
\begin{aligned}
& R e \frac{Z_{\perp}}{n}=\frac{Z_{0}^{2} C}{2 \pi c^{2} \mu_{0}^{2}} F(f) \quad \Omega / m \\
& I m \frac{Z_{\perp}}{n}=\frac{Z_{0}^{2} C}{2 \pi c^{2} \mu_{0}^{2}} G(f) \quad \Omega / m
\end{aligned}
$$

where $Z_{0}^{2} C / 2 \pi c^{2} \mu_{0}^{2} \equiv 31.111$ for the Booster and $G(f), F(f)$ will be called universal frequency functions for the real and imaginary parts of the impedance. Thus, once $F(f)$ and $G(f)$ are known for a given $f$ (see tabulation in next section), the longitudinal and transverse impedances of the kickers are readily evaluated for a given $\beta$ and $x$ by using equations (6) - (11). This will be expanded upon in the next section.

\section{Results}

All curves in Figures (1) - (6) were calculated using $x=.002 m Z_{s}=14 \Omega$, and for proton top energy $(\beta=.9230)$. In figures $(1)$ and $(2)$, the longitudinal and transverse 
impedances for the injection kickers are shown. Figures (3) - (4) show these quantities for the ejection kickers, and in figures (5) - (6) the total longitudinal and transverse impedances are shown for all kickers. The total was evaluated by adding the individual impedances in series.

From figures (5) - (6), we obtain the total longitudinal kicker impedance of $\left|Z_{/ /} / n\right|_{\sim}^{<} 0.042 \Omega$, and the total transverse kicker impedance of $\left|Z_{\perp} / n\right|_{\sim}^{<} 0.37 \mathrm{~m} \Omega / \mathrm{m}$ (calculated for $\left.Z_{\mathrm{a}}=14 \Omega, \beta=.9230, x=2 \mathrm{~mm}\right)$. Comparing these numbers with the values for the space charge ${ }^{2}$, where $\left|Z_{/ /}^{S C} / n\right|_{\sim}^{<} 136 \Omega$ (extraction) and $\left|Z_{\perp}^{S C}\right| \approx 14 M \Omega / m$ (extraction), we see that the overall contribution from the kickers is expected to be quite small.

The numbers quoted above for the impedance assume a reasonable value for the beam orbit offset $x$, and take the value of $\beta$ that corresponds to the maximum value for protons. For different values of $x$ or $\beta$, the following table for $F(f)$ and $G(f)$ should be used:

Table 1

Tabulation of Universal Frequency Functions $\mathbf{F}(f), \mathbf{G}(f)$ defined in Section II

\begin{tabular}{cccccc}
$\mathrm{F}(\mathrm{f})$ & $\mathrm{G}(\mathrm{f})$ & $\mathrm{f}(\mathrm{MHz})$ & $\mathrm{F}(\mathrm{f})$ & $\mathrm{G}(\mathrm{f})$ & $\mathrm{f}(\mathrm{MHz})$ \\
\hline 0 & 0 & 0 & 1708.4 & 10635 & 25 \\
2049 & 304.65 & 1 & 1632.7 & 10573 & 26 \\
3891 & 1179.2 & 2 & 1621.7 & 10536 & 27 \\
5352.7 & 2511.2 & 3 & 1648.1 & 10552 & 28 \\
6311.8 & 4136.1 & 4 & 1675.1 & 10630 & 29 \\
6720.5 & 5857.8 & 5 & 1667.7 & 10762 & 30 \\
6605.3 & 7484.5 & 6 & 1599.1 & 10921 & 31 \\
6059.5 & 8854.1 & 7 & 1460.3 & 11071 & 32 \\
5224 & 9857.1 & 8 & 1260.8 & 11177 & 33 \\
4262.1 & 10448 & 9 & 1026.5 & 11213 & 34 \\
3331.7 & 10649 & 10 & 792.99 & 11166 & 35 \\
2561.2 & 10533 & 11 & 597.59 & 11044 & 36 \\
2032.4 & 10213 & 12 & 469.93 & 10871 & 37 \\
1772.7 & 9814 & 13 & 425.29 & 10682 & 38 \\
1757.9 & 9449.9 & 14 & 461.03 & 10515 & 39 \\
1923.9 & 9207.2 & 15 & 557.28 & 10403 & 40 \\
2184.4 & 9131.9 & 16 & 681.52 & 10365 & 41 \\
2450.5 & 9227.2 & 17 & 796.08 & 10404 & 42 \\
2648.3 & 9459.9 & 18 & 866.58 & 10506 & 43 \\
2731.6 & 9772.4 & 19 & 869.58 & 10640 & 44 \\
2687.6 & 10099 & 20 & 797.61 & 10771 & 45 \\
2534.1 & 10380 & 21 & 660.6 & 10864 & 46 \\
2311.7 & 10576 & 22 & 483.42 & 10891 & 47 \\
2070.7 & 10673 & 23 & 300.15 & 10841 & 48 \\
1858.5 & 10682 & 24 & 146.54 & 10718 & 49 \\
\hline
\end{tabular}


Table 1 should be used in the following way: to calculate $\operatorname{Re} Z_{/ /} / n$ or $\operatorname{Im} Z_{/ /} / n$ for a given $f$, look up the value of $\mathbf{F}(\mathrm{f})$ or $\mathrm{G}(\mathrm{f})$ and multiply by $\beta x^{2}$ for displacement and velocity of interest. For $R e Z_{\perp} / n$ or $\operatorname{Im} Z_{\perp} / n$ multiply $\mathrm{F}(\mathrm{f})$ or $\mathrm{G}(\mathrm{f})$ by 32.111 , for the given $f$ of interest.

In addition to the above calculation, it is also important to estimate the effect of the ferrite relative permeability $\mu_{f}$, or relative permittivity $\epsilon_{f}$ on the impedance calculation. Repeating the procedure used for the SSC, ${ }^{4}$ the impedance formulas (1) - (4) should be multiplied by a factor $\mathrm{F}$, where

$$
\begin{gathered}
F=e^{-a / s}\left(1+R_{F} / 2 R_{A}\right)^{-1} \\
S=\frac{c}{2 \pi f}\left(\frac{2}{\epsilon_{f} \mu_{f}}\right)^{1 / 2}\left\{\left[1+\left(\frac{c^{2} \mu_{0} \sigma}{2 \pi_{f} \epsilon_{f}}\right)^{2}\right]^{1 / 2}-1\right\}^{-1 / 2} \\
R_{F}=2(a+b) / \mu_{f} \mu_{0} s \ell \\
R_{A}=a / \mu_{o} b \ell
\end{gathered}
$$

Substituting the reasonable values ${ }^{1} \mu_{f}=1600, \epsilon_{f}=12$ and $\sigma^{-1}=10^{7} \Omega m, F$ is calculated to be essentially unity for all but the highest frequencies considered here (when $f \equiv 100 M H z, F=.98)$. This input suggests the physical characteristics of the ferrite on impedance can be neglected.

In conclusion, the proposed physical dimensions of the booster kickers do not contribute a significant amount of the longitudinal or transverse beam impedance, especially when these values are compared directly to the space-charge contributions. The calculations reported here $(x=2 \mathrm{~mm})$ indicate the longitudinal impedance of all the kickers is a factor of $3.24 \times 10^{3}$ smaller than "space-charge" longitudinal impedance, and the transverse impedance is also smaller by a factor of 38 .

\section{References}

1. R. Thomas, Memo to A.G. Ruggiero, March 16, 1989.

2. Booster Conceptual Design Manual, October 1988, p. 2-30 to p. 2-45.

3. G. Nassibian and F. Sacherer, Nucl. Instr. and Meth. 159, 21 (1979).

4. K.Y. Ng, "Fields, Impedances and Structures", Fermilab report No. FN-443, 1987.

5. E. Colton and T.S. Wang, SSC Central Design Group Internal Report, No. SSC-N144.

6. G.R. Lambertson and K.Y. Ng in Workshop on the RHIC Performance, March 21, 1988, BNL 41604, p. 435.

7. A. Soukas, private communication. 


\section{Figure Captions}

Fig. 1 Plot of the total longitudinal impedance of injection kickers in units of $\Omega$. The solid line is $\left|Z_{/ /} / n\right|$, the dotted line is the $\operatorname{Im} Z_{/ /} / n$ and the dot-dashed line is the $\operatorname{Re} Z_{/ /} / n$.

Fig. 2 As figure 1, except the total perpendicular component of the impedance of the injection kickers.

Figs. 3-4 As figures 1 and 2, but for the total longitudinal and transverse impedance respectively of the ejection kickers.

Figs. 5-6 As figures 1 and 2, but for the summed total longitudinal and transverse impedance respectively of the kickers. 


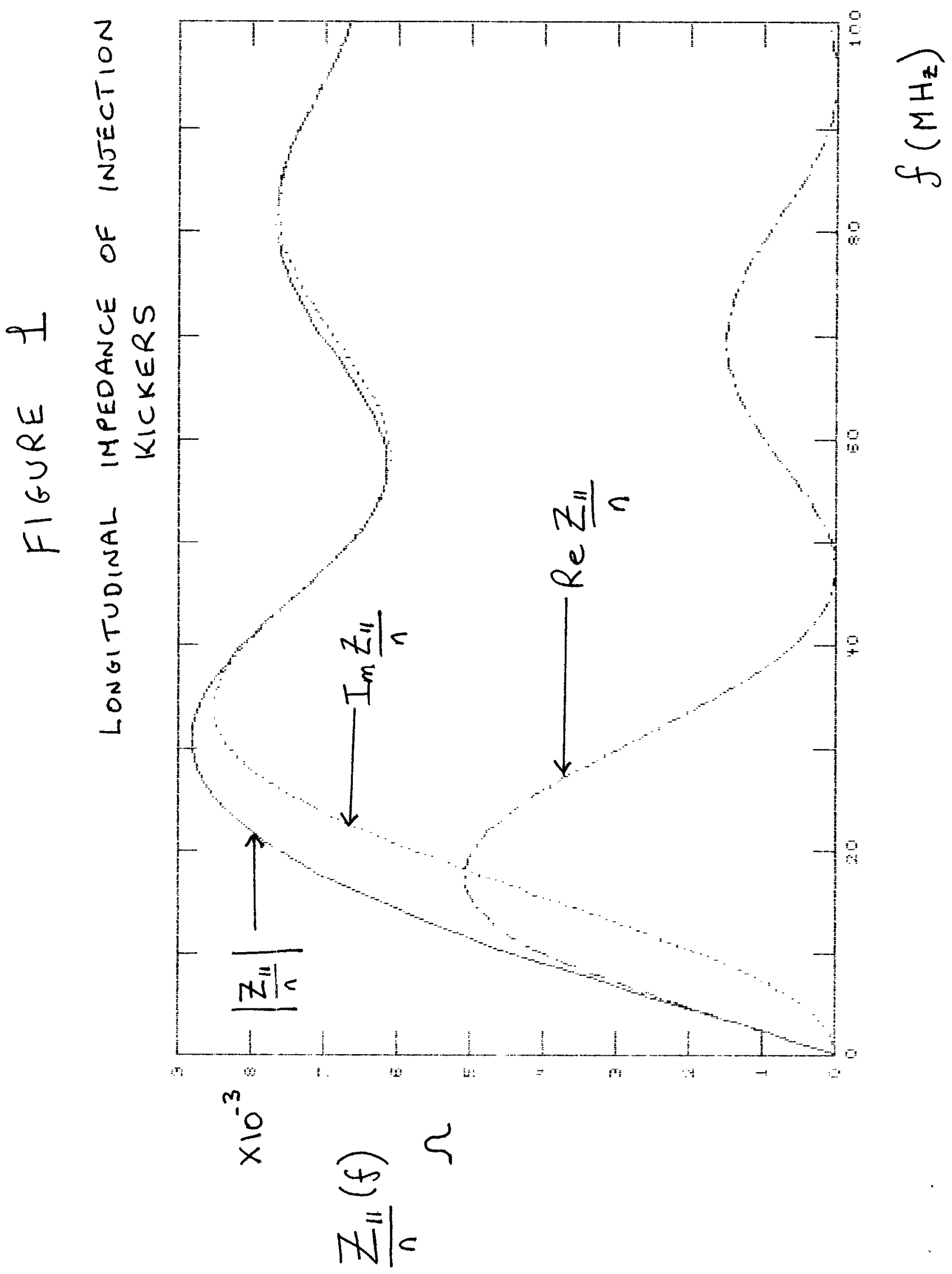




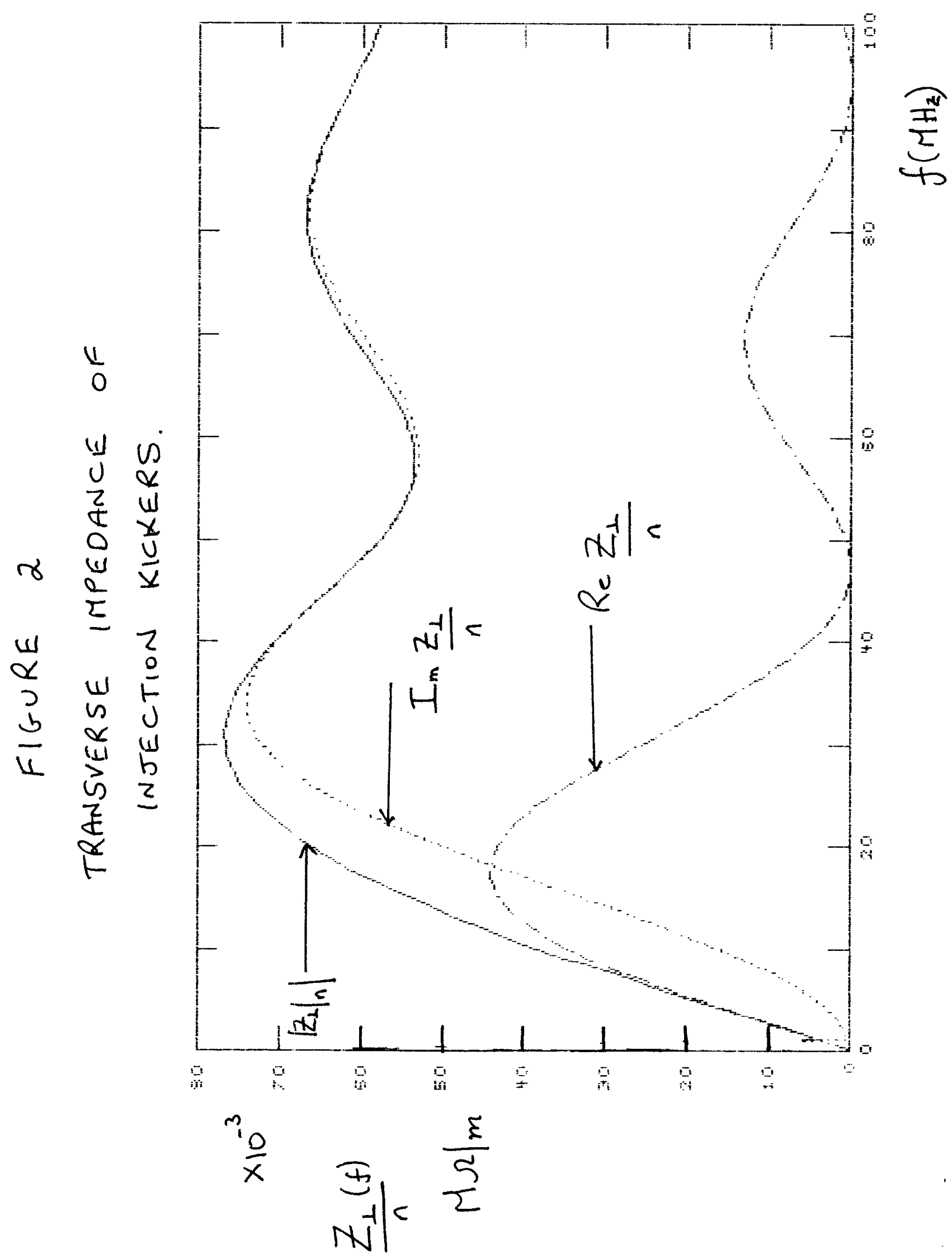




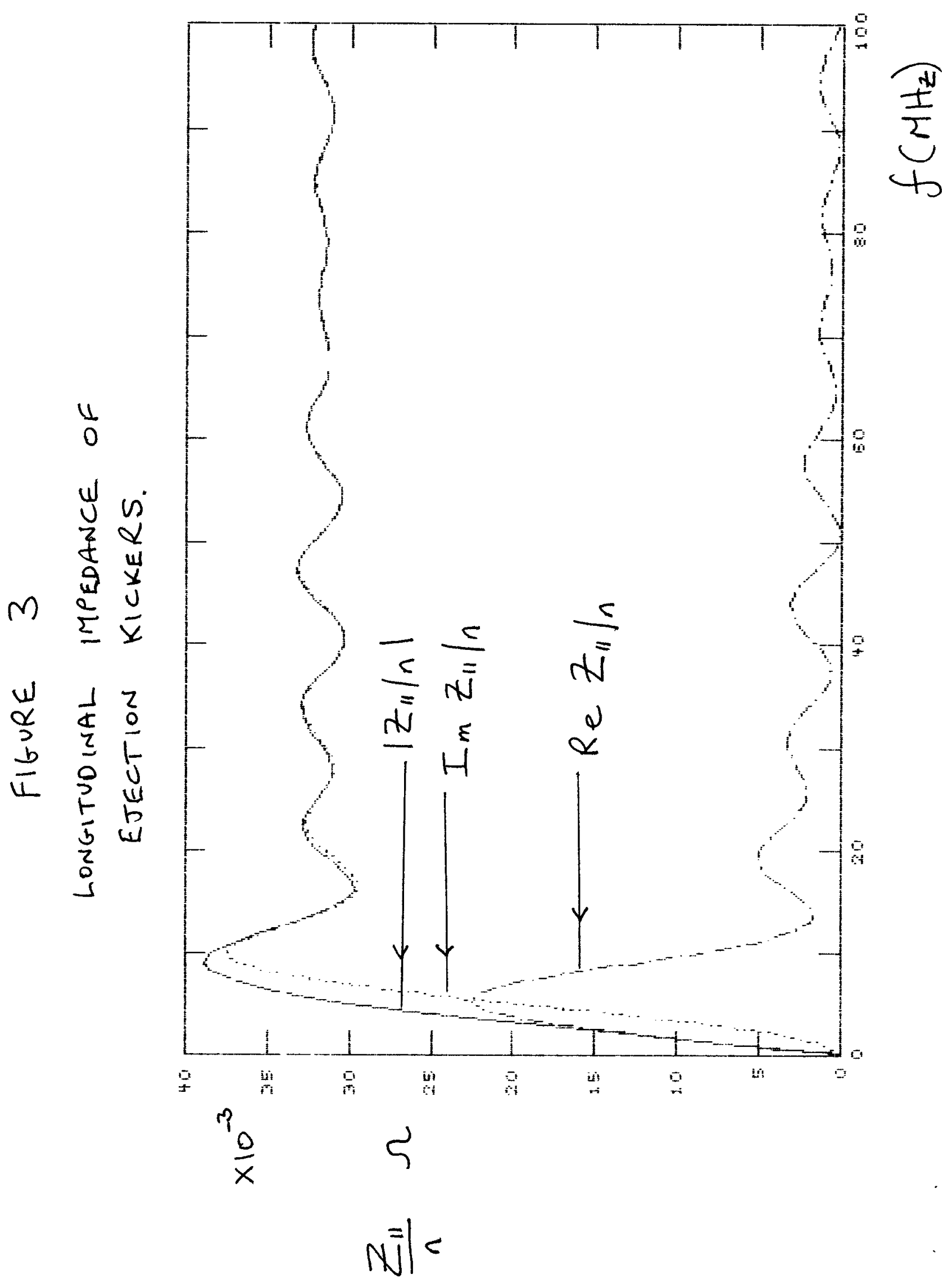




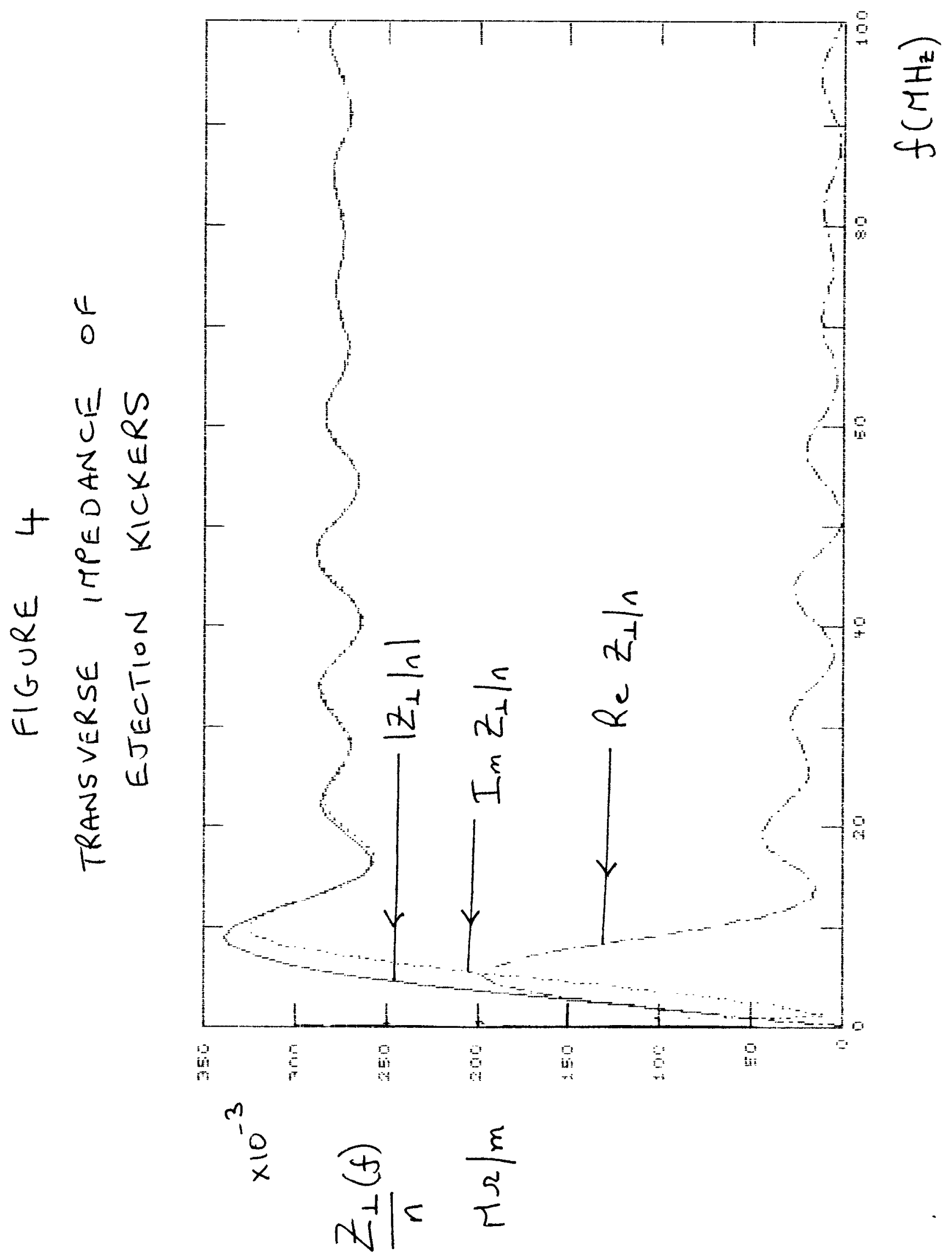




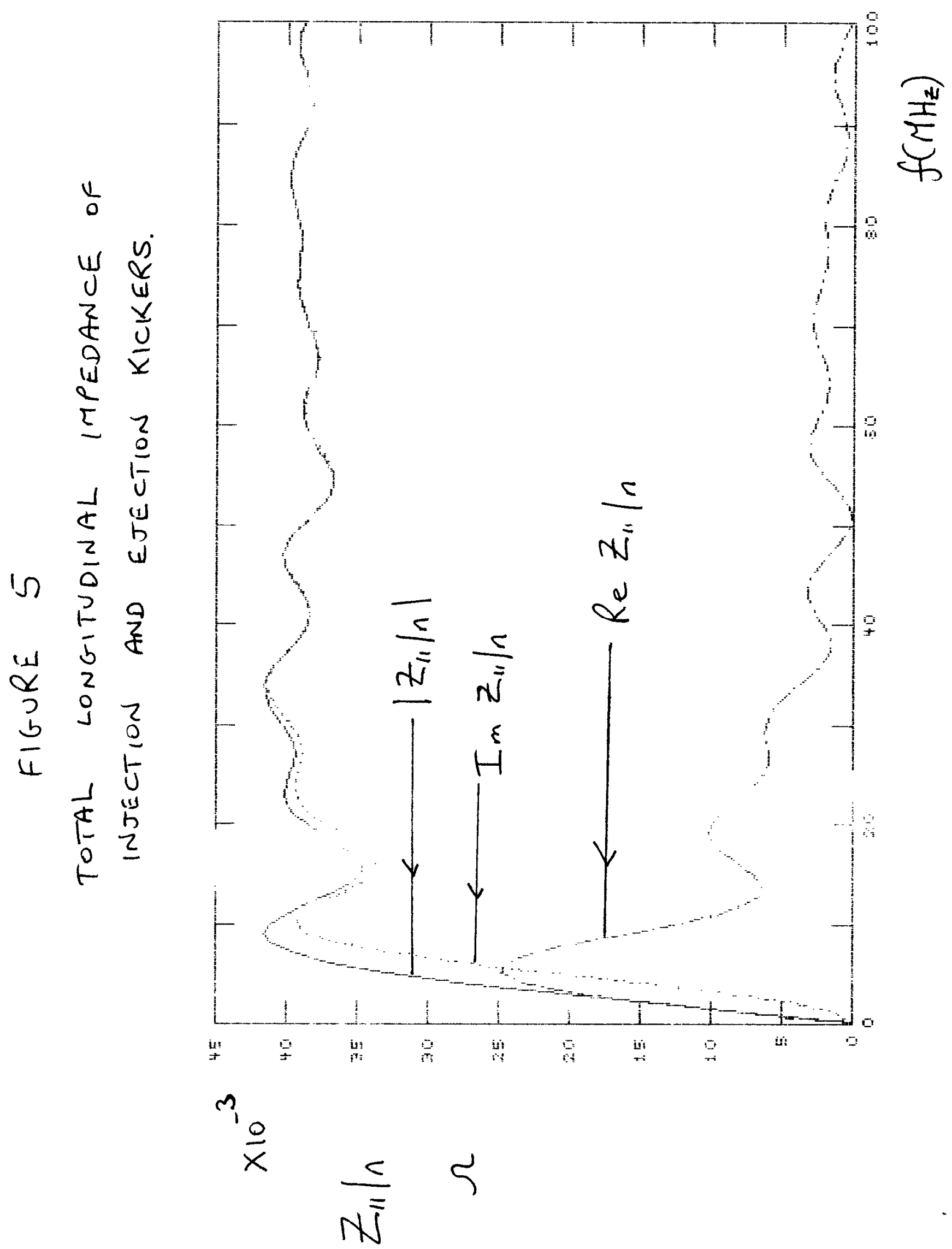




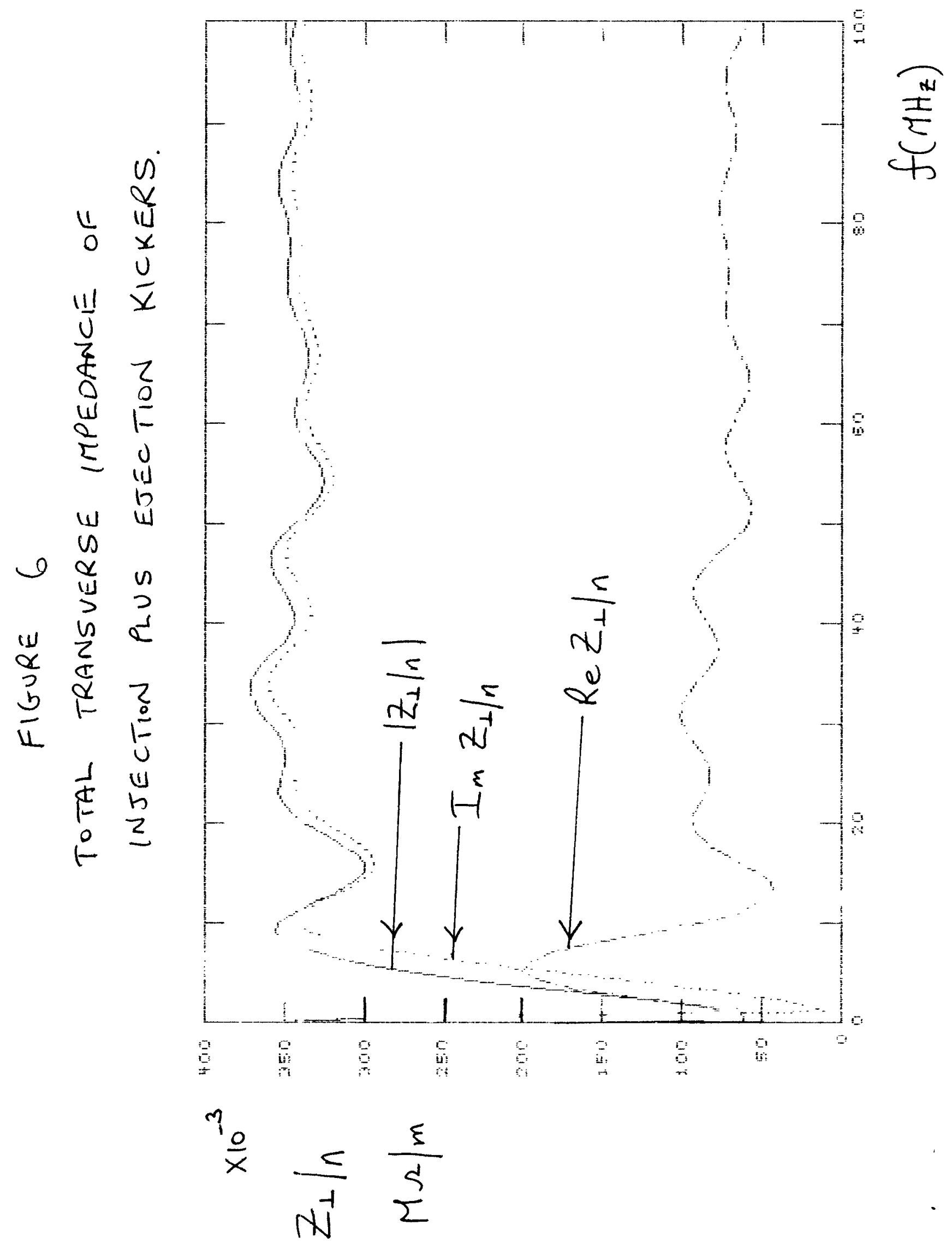

